\title{
Dual Nature of Improper Ferroelectricity in a Magnetoelectric Multiferroic
}

\author{
S. Picozzi, ${ }^{1}$ K. Yamauchi, ${ }^{1}$ B. Sanyal, ${ }^{2}$ I. A. Sergienko, ${ }^{3,4}$ and E. Dagotto ${ }^{3,4}$ \\ ${ }^{1}$ Consiglio Nazionale delle Ricerche - Istituto Nazionale di Fisica della \\ Materia (CNR-INFM), CASTI Regional Lab., 67010 L'Aquila, Italy \\ ${ }^{2}$ Theoretical Magnetism Group, Department of Physics, Uppsala University, Box-530, SE-75121, Sweden \\ ${ }^{3}$ Materials Science 86 Technology Division, Oak Ridge National Laboratory, Oak Ridge, TN 37831, USA \\ ${ }^{4}$ Dept. of Physics $\&$ Astronomy, The University of Tennessee, Knoxville, TN 37996, USA
}

\begin{abstract}
Using first principles calculations, we study the microscopic origin of ferroelectricity (FE) induced by magnetic order in the orthorhombic $\mathrm{HoMnO}_{3}$. We obtain the largest ferroelectric polarization observed in the whole class of improper magnetic ferroelectrics to date. We find that the two proposed mechanisms for FE in multiferroics, lattice- and electronic-based, are simultaneously active in this compound: a large portion of the ferroelectric polarization arises due to quantum-mechanical effects of electron orbital polarization, in addition to the conventional polar atomic displacements. An interesting mechanism for switching the magnetoelectric domains by an electric field via a $180^{\circ}$ coherent rotation of $\mathrm{Mn}$ spins is also proposed.
\end{abstract}

PACS numbers: 75.80.+q, 75.50.Ee, 77.80.-e, 75.47.Lx

Magnetoelectric materials owing their improper ferroelectric (FE) order to symmetry breaking magnetic structures are drawing enormous recent interest [1, 2, 3]. One of the fundamental problems in this area is the understanding of the microscopic origin of their electric polarization. Two basic mechanisms have been proposed in model studies. According to one of them, magnetic ordering results in the hybridization of electronic orbitals producing a polar charge distribution [4, 5]. The other, more conventional approach, views the displacements of ions from their centrosymmetric positions as the primary source of the polarization [6, 7, [8]. Extensive experimental studies have not been able to distinguish between the two possibilities due to very small values of the polarization $P$ found in this class of multiferroics, such as $P<0.1 \mu \mathrm{C} / \mathrm{cm}^{2}$ in $\mathrm{TbMnO}_{3}$ and $\mathrm{TbMn}_{2} \mathrm{O}_{5}$ [1, 2].

In a quest for higher $P$, a recent model Hamiltonian study concentrated on the collinear antiferromagnetic-E (AFM-E) spin configuration, where ferromagnetic zigzag spin-chains in the $\mathrm{MnO}_{2}$ planes are antiferromagnetically coupled with respect to both adjacent in-plane chains (see Fig. 1a) and out-of-plane stacked chains, as found in the orthorhombic $\mathrm{HoMnO}_{3}$ and other perovskite compounds [8, 9]. The predicted polarization $P=0.5-12 \mu \mathrm{C} / \mathrm{cm}^{2}$ was much higher than in other improper magnetic ferroelectrics. However, pyroelectric current measurements on bulk polycrystalline samples revealed ferroelectricity in $\mathrm{HoMnO}_{3}$ with $P$ of only less than $2 \mathrm{nC} / \mathrm{cm}^{2}$ [10].

First-principles calculations appear to be well-suited to address the two issues discussed above: (1) they can reveal the dominant mechanism of improper FE polarization in magnetically ordered compounds, since the electronic structure and lattice distortions can be treated simultaneously, and (2) they can solve the theoryexperiment discrepancy for $\mathrm{HoMnO}_{3}$ in the AFM-E phase by clarifying the true value of the electric polarization.

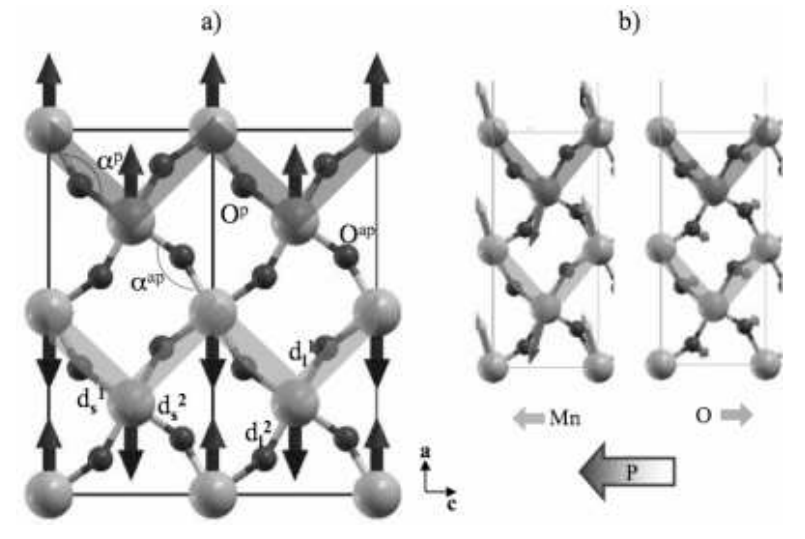

FIG. 1: (Color online) a) The in-plane arrangement of Mn and $\mathrm{O}$ atoms. Arrows denote the direction of spins and antiferromagnetically-coupled zig-zag spin-chains are highlighted by pink and blue shaded areas. Structural parameters reported in Table \are shown. b) Arrows show the directions of the in-plane ionic displacements for Mn (left) and O (right) in the AFM-E phase. The thick arrows at the bottom show the direction of the resulting displacements the $\mathrm{Mn}$ and $\mathrm{O}$ sublattices and $\mathbf{P}$.

We perform simulations based on the generalized gradient approximation (GGA) 11] to density functional (DF) theory using the Vienna Ab-initio Simulation Package (VASP) 12 and the projector-augmented-wave pseudopotentials [13]. Ho $4 f$ electrons were assumed as frozen in the core. The plane wave energy cut-off was set to 500 (400) eV for the collinear (non-collinear) calculations. The Brillouin zone sampling was performed using the $3 \times 4 \times 6$ shell [14]. The GGA+U [15] calculations are performed by applying a Hubbard-like potential for $\mathrm{Mn}$ $d$ states, with $\mathrm{U}$ ranging from 0 to $8 \mathrm{eV}$; accordingly, we chose $\mathrm{J}=0.15 \mathrm{U}$. The Berry phase approach [16, 17] was used to calculate $P$, integrating over six k-point strings parallel to the $c$ axis, each string containing $6 \mathrm{k}$-points. 
Non-collinear calculations were performed according to Ref. [18]. Spin-orbit coupling (SOC) was neglected. As for the structural details, we chose the experimental lattice constants [19] for $\mathrm{HoMnO}_{3}$ for the orthorhombic unit-cell (space group Pnma, $a=5.835 \AA, b=7.361 \AA$ and $c=5.257 \AA$ ) and performed atomic relaxations until the Hellman-Feynman forces were below $0.015 \mathrm{eV} / \AA$.

Due to the small size of the Ho ionic radius, $\mathrm{HoMnO}_{3}$ shows [19] a highly distorted perovskite structure with the Mn-O-Mn $a c$-plane angle $\alpha_{0} \approx 144^{\circ}$. The AFM Etype spin-alignment is stabilized due to this strong distortion of the perovskite structure, as was suggested by previous model [20, 21], and first-principles [22] reports. With the optimized atomic positions obtained after imposing the spin order, we calculated the FE polarization using the point charge model $(\mathrm{PCM})$ with nominal charges. The calculated $P_{\mathrm{PCM}}$ is shown in Fig. 2b as a function of the Hubbard parameter $U . P_{\mathrm{PCM}}(U)$ is a decreasing function, which is consistent with the general ideas of the model calculations [8]. In that model, the polarization appears due to the difference $\left(\alpha_{p}-\alpha_{a p}\right)$ between the Mn-O-Mn angles corresponding to the bonds with parallel $\left(\alpha_{p}\right)$ and antiparallel $\left(\alpha_{a p}\right)$ spins, which is a consequence of the Hund's coupling and virtual electronic hopping. In turn, $U$ characterizes the energy penalty paid for adding an additional electron on a Mn site. Thus, increasing $U$ makes the virtual electron hopping less favorable, which reduces $\alpha_{p}-\alpha_{a p}$ and, ultimately, $P$. Nevertheless, as shown in Fig. 2, the effect of even a very large $U=8 \mathrm{eV}$ is to decrease $P$ by less than half an order of magnitude. As the value of $U$ for $\mathrm{HoMnO}_{3}$ is not known from experiments, we will resort to a parameterfree DF treatment with $U=0$ case from now onwards, noting that the calculated quantities should be trusted at least with respect to their orders of magnitude.

A close look at the geometrically optimized structure in the AFM-E phase reveals a complicated picture of displacements shown in Fig. 10. The Mn atoms displace by $0.04 \AA$ with respect to the initial centrosymmetric Pnma structure. The $a$ and $b$ components of these displacements of different Mn atoms compensate each other, whereas the $c$ components add up to a net displacement of $0.01 \AA$ per Mn atom along the negative direction of the $c$-axis. Similarly, the in-plane $\mathrm{O}$ atoms are displaced on average by $0.02 \AA$ with the resulting $c$-axis displacement of $0.01 \AA$ per atom in the positive direction. Taking into account relatively smaller $c$-axis displacements of interplane $\mathrm{O}$ and Ho atoms we obtain $P_{\mathrm{PCM}}=2.1 \mu \mathrm{C} / \mathrm{cm}^{2}$. Therefore, our calculations with no fitting parameters independently confirm the large electric polarization obtained in the previous model [8]. In this regard, we hope that the experimentally attained $P$ could be improved substantially as it was accomplished for another promising multiferroic $\mathrm{BiFeO}_{3}$ [23, 24].

To have a better understanding of the structural distortions caused by the magnetic order, we also optimize

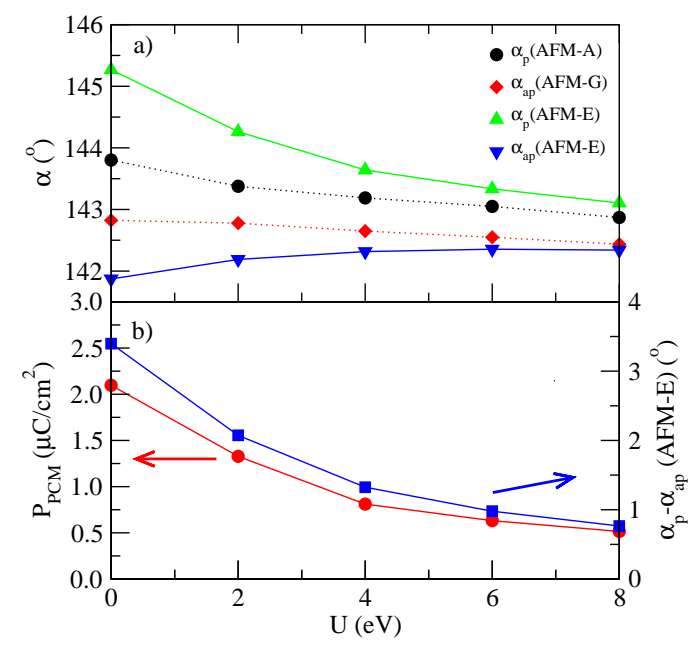

FIG. 2: (Color online) a) Mn-O-Mn angles (in degrees) in the parallel $\left(\alpha_{p}\right)$ and antiparallel $\left(\alpha_{a p}\right)$ Mn spin configurations vs. the Hubbard parameter U. b) Polarization calculated by the point charge model and $\alpha_{p}-\alpha_{a p}$ in the AFM-E phase.

TABLE I: Relevant structural parameters for the AFM-A, G and E spin configurations: Mn-O-Mn angle (in degrees) for parallel $\left(\alpha_{p}\right)$ and anti-parallel $\left(\alpha_{a p}\right)$ Mn spins, large $\left(d_{l}^{1}\right.$ and $\left.d_{l}^{2}\right)$ and small $\left(d_{s}^{1}\right.$ and $\left.d_{s}^{2}\right)$ Mn-O bond-lengths (in $\AA$ ).

\begin{tabular}{|c|c|c|c|c|c|c|}
\hline \hline & $\alpha_{p}$ & $\alpha_{a p}$ & $d_{l}^{1}$ & $d_{l}^{2}$ & $d_{s}^{1}$ & $d_{s}^{2}$ \\
\hline \hline AFM-A & 143.8 & - & 2.20 & 2.20 & 1.93 & 1.93 \\
\hline AFM-G & - & 142.8 & 2.24 & 2.24 & 1.90 & 1.90 \\
\hline AFM-E & 145.3 & 141.9 & 2.25 & 2.18 & 1.92 & 1.92 \\
\hline
\end{tabular}

the structural parameters for the paraelectric AFM-A (all spins in the ac-plane are parallel) and AFM-G (all neighboring spins are antiparallel) phases and compare them

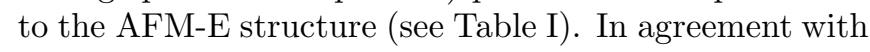
the above discussion, the calculated $\alpha_{p}$ for AFM-A is larger then $\alpha_{a p}$ for AFM-G. However, when both types of bonds (parallel and antiparallel) are present in the AFM-E phase, this difference is even more pronounced, which explains the relatively high $P$. In addition, the $U$-dependence of the angles is shown in Fig. 2a a.

The AFM-E phase shows two different kinds of AFM domains [8], $E_{1}$ and $E_{2}$ (see the left and right insets of Fig. [3a, respectively), which are expected to show opposite polarization, $-P_{c}$ and $P_{c}$. In our calculational unit cell, $E_{1}$ and $E_{2}$ differ in the orientation of half of the spins (see grey highlighted regions in the central inset). Here, we consider a FE-AFM switching path from $-P_{c}\left(E_{1}\right)$ to $P_{c}\left(E_{2}\right)$ via a progressive rotation of the central spins. According to the basic displacement-like mechanism for polarization, we expect $P$ to switch from negative (in $E_{1}$ ) to positive (in $E_{2}$ ) and to vanish when the relative orientation of the central spins with respect to the fixed spins is close to $90^{\circ}$. The $90^{\circ}$ spin-configuration denoted as $\perp$ is shown in the central inset of Fig. 33. More precisely, 
$\perp$ is an example of a spiral magnetic structure similar to that in $\mathrm{TbMnO}_{3}$, but commensurate with the modulation vector $\mathbf{k}=(1 / 2,0,0)$, and which should be $\mathrm{FE}$ with $\mathbf{P}_{\perp}$ along the $c$-axis $[4,6,25,26]$. Based on the macroscopic symmetry considerations 27], the components of the polarization vector $\mathbf{P}$ can be expressed as follows,

$$
P_{c}=\chi_{z}\left(c_{x z} \sin \phi-c_{0} \cos \phi\right), \quad P_{a}=c_{x z}^{\prime} \chi_{x} \sin \phi, \quad P_{b}=0
$$

where $\chi_{x}$ and $\chi_{z}$ are the components of the dielectric susceptibility along the $a$ and $c$-axes, respectively, and $\phi$ is the rotation angle of the central spins. The coefficient $c_{0}$ stems from nonrelativistic interactions, while $c_{x z}$ and $c_{x z}^{\prime}$ originate from the coupling of $P$ to the product of the $a$ and $c$ components of the Mn spins, which has a relativistic origin.

Equations (11) lead to several important conclusions. First, for the commensurate spiral state $\perp(\phi=\pi / 2)$, a longitudinal component $P_{a}$ of the uniform polarization is present in addition to $P_{c}$. Second, $P_{\perp}$ is finite due to purely relativistic effects, in agreement with previous microscopic models [4, 6]. Third, since the relatively small relativistic effects such as SOC are neglected in our computations, we observe that only the $c$ component of the calculated $\mathbf{P}$ is finite for all $\phi$ and $P \propto \cos \phi$, in excellent agreement with the numerical results in Fig. 3b, which are discussed below. Also, in our computations $P_{\perp}=0$, and $\perp$ is taken as the reference paraelectric structure with centrosymmetric positions (csp).

If $\mathrm{FE}$ switching is to occur, as the spin rotation proceeds starting from $E_{1}$, the total energy is expected to increase up to a maximum corresponding to the "paraelectric" state $(\perp)$, and then to decrease again until the $E_{2}$ minimum is reached. Indeed, this happens when we perform the non-collinear calculations by varying $\phi$ between 0 and $180^{\circ}$ with the full optimizations of the internal atomic coordinates for each spin configuration. The calculated total energy of the system as a function of $\phi$ (see Fig 3 a) clearly shows a double-well structure, with the depth of the well of about $8 \mathrm{meV} /$ formula unit (f.u.). Although the exact magnitude of the depth of the well can be affected by computational details and approximations, we expect the feasibility of the magnetoelectric switching by the application of realistic electric fields. The calculated energy barrier is, in fact, smaller than in proper $\mathrm{FE}$ $\mathrm{BaTiO}_{3}(18 \mathrm{meV} /$ f.u. $)$ and $\mathrm{PbTiO}_{3}(200 \mathrm{meV} /$ f.u. $)$, and multiferroic $\mathrm{BaMF}_{4}$ (> $20 \mathrm{meV} /$ f.u.) [28, 29].

The evaluation of $P$ deserves a careful discussion, since it leads to an intriguing outcome. In Fig $3 \mathrm{~b}$, we report the polarization evaluated by the PCM and Berryphase (BP) approaches within the density-functionaltheory (DFT-BP) along the previously mentioned switching path. There is a marked disagreement between the PCM and DFT-BP approaches, therefore suggesting that purely quantum electronic effects are at play in determining the final $P$, similar to the conventional FEs [30]. To

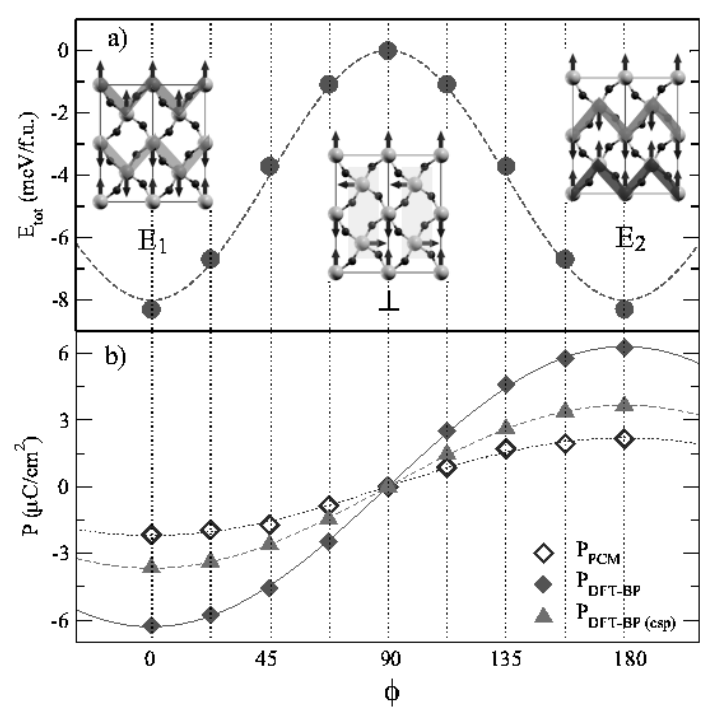

FIG. 3: (Color online) a) Total energy as a function of the orientation of the central spins (see gray highlighted regions in the central $\perp$ spin-configuration) with respect to the spin of the $\mathrm{Mn}$ in the origin. b) Polarization calculated via the PCM (empty blue diamonds) and quantum-mechanically via DFT-BP (filled red diamonds). The pink triangle denotes the values obtained via the DFT-BP approach for centrosymmetric atomic configurations as explained in the text. The lines are fits to $P \propto-\cos \phi$ with constant coefficients.

investigate the purely electronic effects, we calculate $P$ considering the atomic positions of the $\perp$ structure and artificially switching the spin-configuration, without relaxing the lattice degrees of freedom. In this case, due to structural centrosymmetry, there is no contribution from atomic displacements. However, the calculated BP polarization is found to be up to $3.5 \mu \mathrm{C} / \mathrm{cm}^{2}$ (see triangles in Fig. 3b). This large contribution arises solely from the electronic contribution due to symmetry breaking by the AFM-E ordering.

The quantum effects can be quantified further by considering the deviations of the effective Born charges from their nominal valencies. In the $\perp$ case, when each $O$ is coordinated to two Mn atoms with perpendicular spins, we obtain $Z_{\perp}^{*}(\mathrm{Mn})=3.9 e$ and $Z_{\perp}^{*}(\mathrm{O})=-3.1 e$. Along the AFM-FE switching path, the $\mathrm{O}$ ions $\mathrm{O}^{p}$ and $\mathrm{O}^{a p}$ become increasingly different and acquire different Born charges due to the orientation of the Mn spins to which they are bonded. In the extreme points corresponding to AFM-E1 and AFM-E2, we obtain: $Z^{*}(\mathrm{Mn})=3.8 e$, $Z^{*}\left(\mathrm{O}^{a}\right)=-2.6 e$ and $Z^{*}\left(\mathrm{O}^{a p}\right)=-3.5 e$. Note, that in both $\perp$ and E-type spin arrangements, the Born charges are not extremely different from their nominal valence, consistent with a rather ionic nature of the chemical bonds. We note that the two different kinds of $\mathrm{O}$ ions are responsible for ferroelectricity in the case of centrosymmetric positions, where the displacement mechanism is 


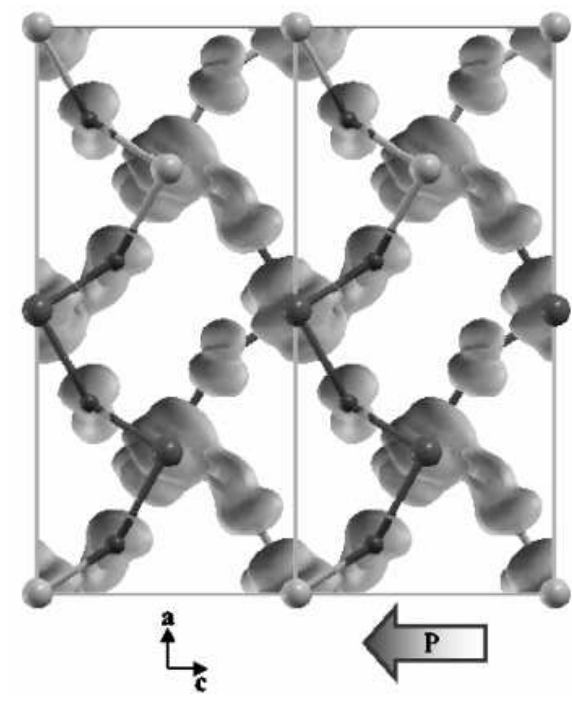

FIG. 4: (Color online) The $a c$-plane charge density isosurface plot in the energy range [-0.8:0] eV (considering the top of the valence band as zero of the energy scale) for fully relaxed positions in the AFM-E1 spin configuration. Blue (green) balls denote Mn atoms with up (down) spins.

switched off. When atomic relaxation is allowed, all the atoms (including displaced $\mathrm{Mn}$ ) contribute to the final $\mathrm{FE}$ polarization. The inequivalence of $\mathrm{O}^{a}$ and $\mathrm{O}^{a p}$ is further confirmed by the charge density plot for the fullyrelaxed structure, corresponding to the energy range of hybridized $\mathrm{Mn} e_{g}$ and $\mathrm{O} p$ orbitals located just below the valence band maximum, as shown in Fig. 4. In addition to the expected G-like orbital ordering [9, 20], Fig. 4 clearly demonstrates that there is a strong asymmetry in the charge distribution between the two $\mathrm{O}$ ions. Moreover, focusing on $\mathrm{O}^{a p}$, the charge seems to favor the short Mn-O bond compared to the long bond. This suggests that the polar charge distribution and related wave-functions are due to a delicate combination of the Jahn-Teller effect and symmetry-breaking magnetic ordering.

In summary, our first-principles results show that, in the AFM-E-type $\mathrm{HoMnO}_{3}$, the symmetry-induced inequivalence of the in-plane Mn-O-Mn configurations for parallel and antiparallel spins is an efficient mechanism in driving a considerable ferroelectric polarization. The calculated total polarization of the AFM-E phase, $P \approx$ $6 \mu \mathrm{C} / \mathrm{cm}^{2}$ is consistent with the previously obtained theoretical estimates [8]. In addition to the displacement mechanism, we find a larger but comparable contribution arising from a purely electronic quantum effect of orbital polarization. The finite ferroelectric polarization, even with a centrosymmetric atomic arrangement, is an unambiguous indication of a magnetism-induced electronic mechanism at play. Also, a magnetoelectric domain switching path is proposed, in which the reversal of polarity of the applied electric field induces a $180^{\circ}$-flip of selected spins. Although we have focused on the case of $\mathrm{HoMnO}_{3}$ as example, we believe our results concerning the dual nature of ferroelectricity as arising from a symmetry breaking induced by the magnetic order should have a wider validity for improper magnetic ferroelectrics. Our findings suggest that the interpretation of experiments, as well as model calculations, should take into account both the lattice and electronic mechanisms of improper ferroelectricity in multiferroics.

Computational support from Barcelona Supercomputing Center and Swedish National Infrastructure for Computing (SNIC) is acknowledged. Research at ORNL is sponsored by the Division of Materials Sciences and Engineering, Office of Basic Energy Sciences, U.S. Department of Energy, under Contract No. DE-AC0500OR22725 with Oak Ridge National Laboratory, managed and operated by UT-Battelle, LLC. I.S. and E.D. are supported in part by NSF Grant No. DMR-0443144.

[1] T. Kimura, T. Goto, H. Shintani, K. Ishizaka, T. Arima, and Y. Tokura, Nature 426, 55 (2003).

[2] N. Hur, S. Park, P. A. Sharma, J.S. Ahn, S. Guha, and S. W. Cheong, Nature 429, 392 (2004).

[3] S.W.Cheong and M.Mostovoy, Nature Mater. 6, 13 (2007).

[4] H.Katsura, N.Nagaosa, and A.V. Balatsky, Phys. Rev. Lett. 95, 057205 (2005).

[5] C.Jia, S.Onoda, N. Nagaosa, and J.H.Han, cond-mat/0701614.

[6] I.A.Sergienko and E.Dagotto, Phys. Rev. B 73, 094434 (2006).

[7] N. Aliouane, D.N.Argyriou, J. Strempfer, I. Zegkinoglou, S. Landsgesell, and M. Zimmermann, Phys. Rev. B 73, 020102(R) (2006).

[8] I.A. Sergienko, C. Şen, and E. Dagotto, Phys. Rev. Lett. 97, 227204 (2006).

[9] A. Muñoz, M.T.Casáis, J.A.Alonso, M.J.Martínez-Lope, J.L.Martínez, and M.T.Fernández-Díaz, Inorg. Chem. 40, 1020 (2001).

[10] B.Lorenz, Y.Q.Wang, and C.W.Chu, cond-mat/0608195.

[11] J.P.Perdew, K.Burke, and M. Ernzerhof, Phys. Rev. Lett. 77, 3865 (1996)

[12] G.Kresse and J.Furthmüller, Phys.Rev.B 54, 11169 (1996).

[13] G.Kresse and G. Joubert, Phys. Rev. B 59, 1758 (1999).

[14] H.J.Monkhorst and J.D.Pack, Phys.Rev.B 13, 5188 (1976).

[15] V.I.Anisimov, F.Aryasetiawan and A.I.Lichtenstein, J. Phys.: Cond. Mat. 9, 767 (1997).

[16] R.D.King-Smith and D.Vanderbilt, Phys. Rev. B 47, 1651 (1993).

[17] R. Resta, Rev. Mod. Phys 66, 899 (1994).

[18] D. Hobbs, G. Kresse and J.Hafner, Phys. Rev. B 62, 11556 (2000).

[19] A.J.Alonso, M.J.Martinez-Lope, M.T. Casais, and M.T.Fernandez-Diaz, Inorg. Chem.39, 917 (2000).

[20] T.Hotta, M.Moraghebi, A. Feiguin, A.Moreo, S.Yunoki, and E.Dagotto, Phys. Rev. Lett. 90, 247203 (2003). 
[21] T. Kimura, S.Ishihara, H. Shintani, T. Arima, K.T.Takahashi, K.Ishizaka, and Y.Tokura, Phys. Rev. B 68, 060403(R) (2003).

[22] S.Picozzi, K. Yamauchi, G. Bihlmayer, and S. Bluegel, Phys. Rev. B 74, 094402 (2006).

[23] J.B.Neaton, C.Ederer, U.V.Waghmare, N.A.Spaldin, and K.M.Rabe, Phys. Rev. B 71, 014113 (2005).

[24] R. Ramesh and N.A.Spaldin, Nature Mater. 6, 21 (2007).

[25] M.Mostovoy, Phys. Rev. Lett. 96, 067601 (2006).

[26] M.Kenzelmann, A.B.Harris, S.Jonas, C.Broholm, J.Schefer, S.B.Kim, C.L.Zhang, S.W.Cheong, O.P.Vajk, and W.J.Lynn, Phys. Rev. Lett. 95, 087206 (2006).

[27] See EPAPS Doc. No. XXX for the explicit derivation.
[28] R.E.Cohen, Nature 358, 136 (1992).

[29] C.Ederer and N.A.Spaldin, Phys. Rev. B 74, 024102 (2006).

[30] W.Zhong, R.D.King-Smith, and D. Vanderbilt, Phys. Rev. Lett. 72, 3618 (1994).

[31] L.D. Landau and E.M. Lifshitz, Statistical Physics, Part $I$ (Pergamon Press, Oxford, 1980).

[32] T. Hahn, (ed.) International Tables for Crystallography 5th edn, Vol. A (Kluwer, Dordrecht, 2002).

[33] J.C. Tolédano and P. Tolédano The Landau Theory of Phase Transitions (World Scientific, Singapore, 1987).

\section{Auxiliary Material}

Here we give a brief derivation of selected results of the Landau theory theory of phase transitions 31] applied to the AFM-E order and its coupling to ferroelectricity. Here we work in the Pnma (\#62) space group setting, which is related to Pbnm used in Ref. 8 via a different choice of the orthorhombic axes 32]. We also place the origin in the $\mathrm{Mn}$ position. The order parameter of the magnetic ordering $\left(\mathbf{E}_{1}, \mathbf{E}_{2}\right)$ is written as a linear combination of Mn spins $\mathbf{S}_{i}$, where $i=1, \ldots, 8$ denotes the Mn atoms occupying the following positions in the magnetic unit cell, corresponding to the modulation vector $\mathbf{k}=(1 / 2,0,0)$ :

$$
1:(000), \quad 2:\left(\frac{1}{2} 0 \frac{1}{2}\right), \quad 3:(100), \quad 4:\left(\frac{3}{2} 0 \frac{1}{2}\right), \quad 5:\left(0 \frac{1}{2} 0\right), \quad 6:\left(\frac{1}{2} \frac{1}{2} \frac{1}{2}\right), \quad 7:\left(1 \frac{1}{2} 0\right), \quad 8:\left(\frac{3}{2} \frac{1}{2} \frac{1}{2}\right),
$$

The order parameter is obtained as $[8]$

$$
\begin{aligned}
& \mathbf{E}_{1}=\mathbf{S}_{1}-\mathbf{S}_{2}-\mathbf{S}_{3}+\mathbf{S}_{4}-\mathbf{S}_{5}+\mathbf{S}_{6}+\mathbf{S}_{7}-\mathbf{S}_{8} \\
& \mathbf{E}_{2}=\mathbf{S}_{1}+\mathbf{S}_{2}-\mathbf{S}_{3}-\mathbf{S}_{4}-\mathbf{S}_{5}-\mathbf{S}_{6}+\mathbf{S}_{7}+\mathbf{S}_{8} .
\end{aligned}
$$

The components of $\mathbf{E}_{1}$ and $\mathbf{E}_{2}$ transform according to the rules given in Table $\amalg$ where the transformation rules for electric polarization $\mathbf{P}$ are also summarized. Here, we analyze the possible magnetoelectric coupling terms of the form $P \cdot E^{2}$ in the thermodynamic potential:

$$
\begin{aligned}
F_{\mathrm{me}}= & c_{0} P_{z}\left(\mathbf{E}_{1}^{2}-\mathbf{E}_{2}^{2}\right)+c_{y y} P_{z}\left(E_{1}^{y 2}-E_{2}^{y 2}\right)+c_{z z} P_{z}\left(E_{1}^{z 2}-E_{2}^{z 2}\right)+c_{x z} P_{z}\left(E_{1}^{x} E_{2}^{z}-E_{2}^{x} E_{1}^{z}\right)+c_{x z}^{\prime} P_{x}\left(E_{2}^{z} E_{2}^{x}-E_{1}^{z} E_{1}^{x}\right) \\
& +d_{x y} P_{y}\left(E_{1}^{y} E_{2}^{x}-E_{2}^{y} E_{1}^{x}\right)+d_{z y} P_{y}\left(E_{1}^{y} E_{1}^{z}-E_{2}^{y} E_{2}^{z}\right) .
\end{aligned}
$$

Clearly, only the first term has a nonrelativistic origin since $\left(\mathbf{E}_{1}^{2}-\mathbf{E}_{2}^{2}\right)$ is a linear combination of the terms $\left(\mathbf{S}_{i} \cdot \mathbf{S}_{k}\right)$.

Considering Eq. (3) together with the usual dielectric energy $F_{\mathrm{de}}=1 / 2\left(\chi_{x}^{-1} P_{x}^{2}+\chi_{y}^{-1} P_{y}^{2}+\chi_{z}^{-1} P_{z}^{2}\right)$, we obtain that $P_{y}=0$ if $E_{1}^{y}=E_{2}^{y}=0$, which always holds in our case. The other two components of $\mathbf{P}$ are given as

$$
P_{x}=-\chi_{x} c_{x z}^{\prime}\left(E_{2}^{z} E_{2}^{x}-E_{1}^{z} E_{1}^{x}\right), \quad P_{z}=-\chi_{z}\left[c_{0}\left(\mathbf{E}_{1}^{2}-\mathbf{E}_{2}^{2}\right)+c_{z z}\left(E_{1}^{z 2}-E_{2}^{z 2}\right)+c_{x z}\left(E_{1}^{x} E_{2}^{z}-E_{2}^{x} E_{1}^{z}\right)\right] .
$$

As is proposed in the main text, the switching of the magnetoelectric domain is accomplished by rotating the spins $\mathbf{S}_{2}, \mathbf{S}_{4}, \mathbf{S}_{6}$, and $\mathbf{S}_{8}$ in the $x z$-plane. Thus, we set

$$
\mathbf{S}_{1}=-\mathbf{S}_{3}=-\mathbf{S}_{5}=\mathbf{S}_{7}=S(1,0,0), \quad-\mathbf{S}_{2}=\mathbf{S}_{4}=\mathbf{S}_{6}=-\mathbf{S}_{8}=S(\cos \phi, 0, \sin \phi),
$$

Using Eqs. (21) and (44), it is easy to verify that $\phi=0$ and $\phi=\pi$ correspond to the domains $E_{1}^{x}\left(-P_{z}\right)$ and $E_{2}^{x}\left(+P_{z}\right)$, respectively. Furthermore, we obtain,

$$
P_{x}=\chi_{x} 32 S^{2} c_{x z}^{\prime} \sin \phi, \quad P_{z}=\chi_{z}\left(32 S^{2} c_{x z} \sin \phi-64 S^{2} c_{0} \cos \phi\right),
$$

which are equivalent to the expressions given in the main text with the numerical constants and $S^{2}$ adsorbed in the coefficients.

As a by-product of the present analysis we also find that the following term is allowed in the thermodynamic potential:

$$
F_{\mathrm{L}}=\frac{\lambda}{V} \int\left(E_{1}^{x} \partial_{x} E_{2}^{x}-E_{2}^{x} \partial_{x} E_{1}^{x}\right) d V
$$


TABLE II: Matrices of the generators of space group Pnma in the representations spanned by $\mathbf{E}_{1}, \mathbf{E}_{2}$, and $\mathbf{P}$. The space group elements are denoted $(r \mid h k l)$, where $r$ is the identity operation 1 , two-fold rotation $2_{a, c}$, inversion $I$, or time reversal $1^{\prime}$ followed by the translation $\boldsymbol{\tau}=h \mathbf{a}+k \mathbf{b}+l \mathbf{c}$.

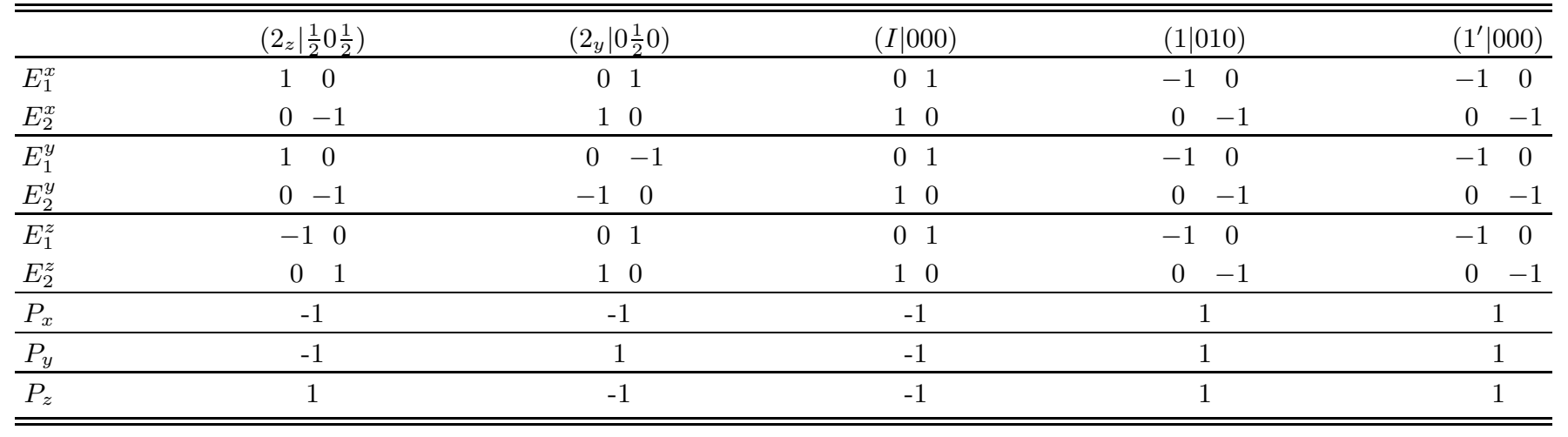

This Lifshitz [31, 33] term leads to the following typical series of phase transition when the temperature is lowered: Para-phase $\rightarrow$ Incommensurate phase $\rightarrow$ "Locked-in" commensurate phase [33], which was indeed observed in $\mathrm{HoMnO}_{3}$ [9]. It can be shown[33] that both $E_{1}^{x}$ and $E_{2}^{x}$ are modulated with $\mathbf{k}(T)=(1 / 2+\delta k(T), 0,0)$ in the AFM-Incommensurate phase. Taking into account the magnetoelectric interactions (3), this leads to a vanishing macroscopic polarization in the incommensurate phase in agreement with the experiment [10]. 